\title{
Serpent: A New Block Cipher Proposal
}

\author{
Eli Biham ${ }^{1}$, Ross Anderson ${ }^{2}$, and Lars Knudsen ${ }^{3}$ \\ 1 Technion, Haifa, Israel; biham@cs.technion.ac.il \\ 2 Cambridge University, England; rja14@cl.cam.ac.uk \\ 3 University of Bergen, Norway; lars.knudsen@ii.uib.no
}

\begin{abstract}
We propose a new block cipher as a candidate for the Advanced Encryption Standard. Its design is highly conservative, yet still allows a very efficient implementation. It uses the well-understood DES S-boxes in a new structure that simultaneously allows a more rapid avalanche, a more efficient bitslice implementation, and an easy analysis that enables us to demonstrate its security against all known types of attack. With a 128-bit block size and a 256-bit key, it is almost as fast as DES on a wide range of platforms, yet conjectured to be at least as secure as three-key triple-DES.
\end{abstract}

\section{Introduction}

For many applications, the Data Encryption Standard algorithm is nearing the end of its useful life. Its 56-bit key is too small, as shown by a recent distributed key search exercise [21]. Although triple-DES can solve the key length problem, the DES algorithm was also designed primarily for hardware encryption, yet the great majority of applications that use it today implement it in software, where it is relatively inefficient.

For these reasons, the US National Institute of Standards and Technology has issued a call for a successor algorithm, to be called the Advanced Encryption Standard or AES. The essential requirement is that AES should be both faster and more secure than triple DES; specifically, it should have a 128 bit block length and a key length of 256 bits (though keys of 128 and 192 bits must also be supported).

In this paper, we present a candidate for AES. Our design philosophy has been highly conservative; we did not feel it appropriate to use novel and untested ideas in a cipher which, if accepted after a short review period, will be used to protect enormous volumes of financial transactions, health records and government information over a period of decades.

We therefore decided to use the S-boxes from DES, which have been subjected to intense study over many years and whose properties are thus well understood, in a new structure which is optimized for efficient implementation on modern processors while simultaneously allowing us to apply the extensive analysis already done on DES. As a result, we can show that our design resists all known attacks, including those based on both differential [7] and linear [20] techniques. 
We propose several variants of the cipher, which we have tentatively named Serpent. The primary variant is a 32-round cipher which we believe to be as secure as three-key triple-DES, but which is only slightly slower than DES when implemented in $\mathrm{C}$ on a Pentium (in some assembly languages it may be faster than DES). It is an SP-network operating on four 32-bit words, thus giving a block size of 128 bits.

The additional variants have increased block sizes. The block size can be doubled to 256 bits either by increasing the word size from 32 to 64 bits (which will be well suited to the new generation of 64-bit processors), or by using the round function in a Feistel construction. These two variants can be combined to give a cipher with 512-bit blocks.

At this stage all the variants are still tentative. We are still working on improvements and analysis. As usual in this field, we encourage interested parties to analyze the cipher, inform us of any weakness, and pass on any remarks or suggestions for improvements.

All values used in the ciphers are represented in little-endian, including the bit order (0-31 in 32-bit words, or 0-127 in the full 128-bit blocks), and the order of words in the block. Thus bit 0 is the least significant bit, and word 0 is the least significant word. The notation is important, as there are two equivalent representations of Serpent: a standard representation and a bitslice representation.

\section{The Cipher}

The main variant of our cipher encrypts a 128-bit plaintext $P$ to a 128-bit ciphertext $C$ in $r$ rounds under the control of $r+1128$-bit subkeys $\hat{K}_{0}, \ldots, \hat{K}_{r}$. (We have chosen $r=32$ as the default, and will henceforth replace $r$ by 32 in order to make the description of the cipher more readable.)

The cipher is an SP-network and consists of:

- an initial permutation $I P$;

- 32 rounds, each consisting of a key mixing operation, a pass through S-boxes, and (in all but the last round) a linear transformation. In the last round, this linear transformation is replaced by an additional key mixing operation;

- a final permutation $F P$.

The initial and final permutations do not have any cryptographic significance. They are used to simplify an optimized implementation of the cipher, which is described in the next section, and to improve its computational efficiency. Both these two permutations and the linear transformation are specified in the appendix; their design principles will be made clear in the next section.

We use the following notation. The initial permutation $I P$ is applied to the plaintext $P$ giving $\hat{B}_{0}$, which is the input to the first round. The rounds are numbered from 0 to 31 , where the first round is round 0 and the last is round 31 . The output of the first round (round 0 ) is $\hat{B}_{1}$, the output of the second round (round 1) is $\hat{B}_{2}$, the output of round $i$ is $\hat{B}_{i+1}$, and so on, until the output of the 
last round (in which the linear transformation is replaced by an additional key mixing) is denoted by $\hat{B}_{32}$. The final permutation $F P$ is now applied to give the ciphertext $C$.

Each round function $R_{i}(i \in\{0, \ldots, 31\}$ uses only a single replicated S-box. For example, $R_{0}$ uses $S_{0}, 32$ copies of which are applied in parallel. Thus the first copy of $S_{0}$ takes bits $0,1,2$ and 3 of $\hat{B}_{0} \oplus \hat{K}_{0}$ as its input and returns as output the first four bits of an intermediate vector; the next copy of $S_{0}$ inputs bits 4-7 of $\hat{B}_{0} \oplus \hat{K}_{0}$ and returns the next four bits of the intermediate vector, and so on. The intermediate vector is then transformed using the linear transformation, giving $\hat{B}_{1}$. Similarly, $R_{1}$ uses 32 copies of $S_{1}$ in parallel on $\hat{B}_{1} \oplus \hat{K}_{1}$ and transforms their output using the linear transformation, giving $\hat{B}_{2}$.

In the last round $R_{31}$, we apply $S_{31}$ on $\hat{B}_{31} \oplus \hat{K}_{31}$, and XOR the result

with $\hat{K}_{32}$ rather than applying the linear transformation. The result $\hat{B}_{32}$ is then permuted by $F P$, giving the ciphertext.

Thus the 32 rounds use 32 different S-boxes each of which maps four input bits to four output bits. Each S-box is used only in one round, in which it is used 32 times in parallel. The 32 S-boxes are chosen as the 32 separate lines of the eight DES S-boxes; thus our $S_{0}$ (used in round 0) is the first line of the DES $S 1$, our $S_{1}$ (used in round 1) is the second line of the DES $S 1$, our $S_{4}$ (used in round 4) is the first line of the DES $S 2$ and so on.

As with DES, the initial permutation is the inverse of the final permutation. Thus the cipher may be formally described by the following equations:

$$
\begin{aligned}
\hat{B}_{0} & =I P(P) \\
\hat{B}_{i+1} & =R_{i}\left(\hat{B}_{i}\right) \\
C & =I P^{-1}\left(\hat{B}_{r}\right)
\end{aligned}
$$

where

$$
\begin{array}{ll}
R_{i}(X)=L\left(\hat{\mathcal{S}}_{i}\left(X \oplus \hat{K}_{i}\right)\right) & i=0, \ldots, r-2 \\
R_{i}(X)=\hat{\mathcal{S}}_{i}\left(X \oplus \hat{K}_{i}\right) \oplus \hat{K}_{r} & i=r-1
\end{array}
$$

where $\hat{\mathcal{S}}_{i}$ is the application of the S-box $S_{i} 32$ times in parallel, and $L$ is the linear transformation.

Although each round of the proposed cipher might seem weaker than a round of DES, we shall see below that their combination overcomes the weakness. The greater speed of each round, and the increased number of rounds, make the cipher both almost as fast as DES and much more secure.

\subsection{Decryption}

Decryption is different from encryption in that the inverse of the S-boxes must be used, as well as the inverse linear transformation and reverse order of the subkeys. 


\section{An Efficient Implementation}

Much of the motivation for the above design will become clear as we consider how to implement the algorithm efficiently. We do this in bitslice mode. For a full description of a bitslice implementation of DES, see [9]; the basic idea is that just as one can use a 1-bit processor to implement an algorithm such as DES by executing a hardware description of it, using a logical instruction to emulate each gate, so one can also use a 32-bit processor to compute 32 different DES blocks in parallel - in effect, using the CPU as a 32-way SIMD machine.

This is much more efficient than the conventional implementation, in which a 32-bit processor is mostly idle as it computes operations on 6 bits, 4 bits, or even single bits. The bitslice approach was used in the recent successful DES key search, in which spare CPU cycles from thousands of machines were volunteered to solve a challenge posed by RSADSI. However the problem with using bitslice techniques for DES encryption (as opposed to keysearch) is that one has to process many blocks in parallel, and although special modes of operation can be designed for this, they are not the modes in common use.

Our cipher has therefore been designed so that all operations can be executed using 32-fold parallelism during the encryption or decryption of a single block. Indeed the bitslice description of the algorithm is much simpler than its conventional description. No initial and final permutations are required, since the initial and final permutations described in the standard implementation above are just the conversions of the data from and to the bitslice representation. We will now present an equivalent description of the algorithm for bitslice implementation.

The cipher consists simply of 32 rounds. The plaintext becomes the first intermediate data $B_{0}=P$, after which the 32 rounds are applied, where each round $i \in\{0, \ldots, 31\}$ consists of three operations:

1. Key Mixing: At each round, a 128-bit subkey $K_{i}$ is exclusive or'ed with the current intermediate data $B_{i}$

2. S Boxes: The 128-bit combination of input and key is considered as four 32-bit words. The S-box, which is implemented as a sequence of logical operations (as it would be in hardware) is applied to these four words, and the result is four output words. The CPU is thus employed to execute the 32 copies of the S-box simultaneously, resulting with $\mathcal{S}_{i}\left(B_{i} \oplus K_{i}\right)$

3. Linear Transformation: The 32 bits in each of the output words are linearly mixed, by

$$
\begin{aligned}
X_{0}, X_{1}, X_{2}, X_{3} & =\mathcal{S}_{i}\left(B_{i} \oplus K_{i}\right) \\
X_{0} & =X_{0}<<<13 \\
X_{2} & =X_{2}<<<3 \\
X_{1} & =X_{1} \oplus X_{0} \oplus X_{2} \\
X_{3} & =X_{3} \oplus X_{2} \oplus\left(X_{0}<<3\right)
\end{aligned}
$$




$$
\begin{aligned}
X_{1} & =X_{1}<<<1 \\
X_{3} & =X_{3}<<<7 \\
X_{0} & =X_{0} \oplus X_{1} \oplus X_{3} \\
X_{2} & =X_{2} \oplus X_{3} \oplus\left(X_{1}<<7\right) \\
X_{0} & =X_{0}<<<5 \\
X_{2} & =X_{2}<<<22 \\
B_{i+1} & =X_{0}, X_{1}, X_{2}, X_{3}
\end{aligned}
$$

where $<<<$ denotes rotation, and $<<$ denotes shift. In the last round, this linear transformation is replaced by an additional key mixing: $B_{r}=S_{r-1}\left(B_{r-1} \oplus\right.$ $\left.K_{r-1}\right) \oplus K_{r}$. Note that at each stage $I P\left(B_{i}\right)=\hat{B}_{i}$, and $I P\left(K_{i}\right)=\hat{K}_{i}$.

The first reason for the choice of linear transformation is to maximize the avalanche effect. The DES S-boxes have the property that a single input bit change will cause two output bits to change; as the difference sets of $\{0,1,3,5$, $7,13,22\}$ modulo 32 have no common member (except one), it follows that a single input bit change will cause a maximal number of bit changes after two and more rounds. The effect is that each plaintext bit, and each round key bit, affect all the data bits after three rounds. Even if an opponent chooses some subkeys and works backwards, it is still the case that each key bit affects each data bit over six rounds. (Some historical information on the design of the above linear transformation is given in the appendix.) The second reason is that it is simple, and can be used in a pipelined processor with a minimum number of pipeline stalls. The third reason is that it was analyzed by programs we developed for investigating block ciphers, and we found bounds on the probabilities of the differential and linear characteristics. These bounds show that this choice suits our needs, although we would like to improve on it.

So we are still considering other, simpler, choices for the linear transformation. One possibility is to adapt an LFSR-like transform of the form $X_{i}=$ $X_{i} \oplus \operatorname{ROL}\left(X_{i-1}, r_{i}\right)$ for $i=1, \ldots, 6$, where the four data words are $X_{0}, \ldots, X_{3}$, the indices of $X$ are taken modulo 4 , and the $r_{i}$ 's are fixed. The problems with such a scheme are that it is hard to pipeline, and that every characteristic can be rotated in all its words and still remain with the same probability. We are still working on other possible linear transformations.

\section{The Key Schedule}

As with the description of the cipher, we can describe the key schedule in either standard or bitslice mode. For reasons of space, we will give the substantive description for the latter case.

Our cipher requires 132 32-bit words of key material. We first expand the user supplied 256 bit key $K$ to 33128 -bit subkeys $K_{0}, \ldots, K_{32}$, in the following way. We write the key $K$ as eight 32 -bit words $w_{-8}, \ldots, w_{-1}$ and expand these to an intermediate key (which we call prekey) $w_{0}, \ldots, w_{131}$ by the following affine recurrence: 


$$
w_{i}=\left(w_{i-8} \oplus w_{i-5} \oplus w_{i-3} \oplus w_{i-1} \oplus \phi \oplus i\right)<<<11
$$

where $\phi$ is the fractional part of the golden ratio $(\sqrt{5}+1) / 2$ or $0 x 9$ e3779b9 in hexadecimal. The underlying polynomial $x^{8}+x^{7}+x^{5}+x^{3}+1$ is primitive, which together with the addition of the round index is chosen to ensure an even distribution of key bits throughout the rounds, and to eliminate weak keys and related keys.

The round keys are now calculated from the prekeys using the S-boxes, again in bitslice mode. The S-box inputs and outputs are taken at a distance of 33 words apart, in order to minimize the key leakage in the event of a differential attack on the last few rounds of the cipher. We use the S-boxes to transform the prekeys $w_{i}$ into words $k_{i}$ of round key by dividing the vector of prekeys into four sections and transforming the $i$ 'th words of each of the four sections using $S_{(r+3-i) \bmod r}$. This can be seen simply for the default case $r=32$ as follows:

$$
\begin{aligned}
\left\{k_{0}, k_{33}, k_{66}, k_{99}\right\} & =S_{3}\left(w_{0}, w_{33}, w_{66}, w_{99}\right) \\
\left\{k_{1}, k_{34}, k_{67}, k_{100}\right\} & =S_{2}\left(w_{1}, w_{34}, w_{67}, w_{100}\right) \\
\ldots & \\
\left\{k_{31}, k_{64}, k_{97}, k_{130}\right\} & =S_{4}\left(w_{31}, w_{64}, w_{97}, w_{130}\right) \\
\left\{k_{32}, k_{65}, k_{98}, k_{131}\right\} & =S_{3}\left(w_{32}, w_{65}, w_{98}, w_{131}\right)
\end{aligned}
$$

We then renumber the 32 -bit values $k_{j}$ as 128 -bit subkeys $K_{i}$ (for $\mathrm{i} \in\{0, \ldots$, r\}) as follows:

$$
K_{i}=\left\{k_{4 i}, k_{4 i+1}, k_{4 i+2}, k_{4 i+3}\right\}
$$

Where we are implementing the algorithm in the form initially described in section 2 above rather than using bitslice operations, we now apply $I P$ to the round key in order to place the key bits in the correct column, i.e., $\hat{K}_{i}=I P\left(K_{i}\right)$.

\section{Security}

As mentioned above, the differential and linear properties of the DES S-boxes are well understood. Our preliminary estimates indicate that the number of known/chosen plaintexts required for either type of attack would exceed $2^{128}$ (they are certainly well over $2^{100}$ and we are working on more accurate bounds). There is thus no indication of any useful shortcut attack; we believe that such an attack would require a new theoretical breakthrough. In any case, it should be noted that regardless of the design of a 128 bit block cipher, it is normally prudent to change keys well before $2^{64}$ blocks have been encrypted, in order to avoid the collision attack of section 5.2 below. This would easily prevent all known shortcut attacks. 
We designed the cipher with a view to reducing or avoiding vulnerabilities arising from the following possible weaknesses and attacks. In our analysis, we use conservative bounds to enable our claims to resist reasonable improvements in the studied attacks. For example, we analyze the cipher using 24-round and 28-round characteristics, shorter by 8 and 4 rounds than the cipher, while the best attack on DES uses characteristics that are shorter by only three rounds. Our estimates of the probabilities of the best characteristics are also very conservative; in practice they should be considerably lower. Therefore, our complexity claims are probably much lower than the real values, and Serpent is expected to be much more secure than we actually claim.

\subsection{Dictionary Attacks}

As the block size is 128 bits, a dictionary attack will require $2^{128}$ different plaintexts to allow the attacker to encrypt or decrypt arbitrary messages under an unknown key. This attack applies to any deterministic block cipher with 128-bit blocks regardless of its design.

\subsection{Modes of Operation}

After encrypting about $2^{64}$ plaintext blocks in the $\mathrm{CBC}$ or $\mathrm{CFB}$ mode, one can expect to find two equal ciphertext blocks. This enables an attacker to compute the exclusive-or of the two corresponding plaintext blocks [18. With progressively more plaintext blocks, plaintext relationships can be discovered with progressively higher probability. This attack applies to any deterministic block cipher with 128-bit blocks regardless of its design.

\subsection{Key-Collision Attacks}

For key size $k$, key collision attacks can be used to forge messages with complexity only $2^{k / 2}$ [5]. Thus, the complexity of forging messages under 128-bit keys is only $2^{64}$, under 192 -bit keys it is $2^{96}$, and under 256 -bit keys it is $2^{128}$. This attack applies to any deterministic block cipher, and depends only on its key size, regardless of its design.

\subsection{Differential Cryptanalysis}

An important fact about Serpent is that any characteristic must have at least one active S-box in each round. At least two active S-boxes are required on average, due to the property that a difference in only one bit in the input causes a difference of at least two bits in the output of each S-box. Therefore, if only one bit differs in the input of some round, then at least two differ in the output, and these two bits affect two distinct S-boxes in the following round, whose output differences affect at least four S-boxes in the following round.

We searched for the best characteristics of this cipher. For this, we made a worst case assumption that all the entries in the difference distribution tables 
have probability $1 / 2$, except the few entries which have only one bit input difference and one bit output difference, which are assumed impossible (probability zero). These bounds are satisfied by all the S-boxes, except for one entry of $S_{30}$, where the maximal value is $10 / 16$ : the highest probabilities in the various S-boxes are 6/16 and 8/16, except in $S_{21}$ in which it is $4 / 16$ and in $S_{30}$ in which it is $10 / 16$. We assume later that round 30 is not approximated by the characteristic anyway. Thus the following results hold independently of the order of the S-boxes used in the cipher, and independently of the choice of the S-boxes, so long as they satisfy these minimal conditions. We searched for the best characteristics with up to seven rounds, and the ones with the highest probabilities are given in Table 1

\begin{tabular}{cccc}
\hline Rounds Differential & \multicolumn{2}{c}{$\begin{array}{c}\text { Linear Probability } \\
(1 / 2 \pm p)\end{array}$} & $p^{-2}$ \\
\hline 1 & $2^{-1}$ & $1 / 2 \pm 6 / 16=1 / 2 \pm 2^{-1.4}$ & $2^{2.8}$ \\
2 & $2^{-3}$ & $1 / 2 \pm(6 / 16)^{3}=1 / 2 \pm 2^{-2.2}$ & $2^{4.4}$ \\
3 & $2^{-7}$ & $1 / 2 \pm(6 / 16)^{8}=1 / 2 \pm 2^{-4.3}$ & $2^{8.6}$ \\
4 & $2^{-13}$ & $1 / 2 \pm(6 / 16)^{14}=1 / 2 \pm 2^{-6.8}$ & $2^{13.6}$ \\
5 & $2^{-21}$ & $1 / 2 \pm(6 / 16)^{20}=1 / 2 \pm 2^{-9.3}$ & $2^{18.6}$ \\
6 & $2^{-29}$ & $1 / 2 \pm(6 / 16)^{27}=1 / 2 \pm 2^{-12.2}$ & $2^{24.4}$ \\
7 & $<2^{-35}$ & $1 / 2 \pm(6 / 16)^{33}=1 / 2 \pm 2^{-13.8}>2^{27.6}$ \\
\hline
\end{tabular}

Table 1. Bounds on the Probabilities of Differential and Linear Characteristics

We can see that the probability of a 6 -round characteristic is bounded by $2^{-29}$. Thus, the probability of a 24-round characteristic is bounded by $2^{-4 \cdot 29}=$ $2^{-116}$. In practice, the probability of the best 24 -round characteristic is expected to be much lower than this. Thus, even if an attacker can implement an 8Rattack, still the attack requires more than $2^{117}$ chosen plaintexts (and again, this is a very conservative estimate). If the attacker can implement only a $4 \mathrm{R}$ attack, using a 28-round characteristic, the probability of the characteristic is bounded by $2^{-4 \cdot 35}=2^{-140}$, and the attack requires more plaintexts than are available. A 3R-attack would require even more plaintexts.

Notice that if the linear transformation had used only rotates, then every characteristic could have 32 equiprobable rotated variants, with all the data words rotated by the same number of bits. This is the reason that we also use shift instructions, which avoid most of these rotated characteristics.

We have bounded the probabilities of characteristics. However, it is both much more important and much more difficult to bound the probabilities of differentials. In order to reduce the probabilities of differentials we have (1) reduced the probabilities of the characteristics, (2) ensured that there are few characteristics with the highest possible probability, and that they cannot be rotated and still remain valid, (3) arranged for characteristics to affect many different bits, so that they cannot easily be unified into differentials. 
We conjecture that the probability of the best 28-round differential is not higher than $2^{-120}$, and that such a differential if it exists would be very hard to find. (Note that for any fixed key there expected to be differentials with probability $2^{-120}$, but averaging over all possible keys reduces this average probability to about $2^{-128}$.)

\subsection{Linear Cryptanalysis}

In linear cryptanalysis, it is possible to find one-bit to one-bit relations of the Sboxes. The probability of these relations are $1 / 2 \pm 2 / 16$. Thus, a 28 -round linear characteristic with only one active S-box in each round would have probability $1 / 2 \pm 2^{27}(2 / 16)^{28}=1 / 2 \pm 2^{-57}$, and that an attack based on such relations would require about $2^{114}$ known plaintexts, if it were possible at all (as the linear transformation assures that in the round following a round with only one active S-box, at least two are active).

More general attacks can use linear characteristics with more than one active S-box in some of the rounds. In this case the probabilities of the S-boxes are bounded by $1 / 2 \pm 6 / 16$. As with differential cryptanalysis, we can bound the probability of characteristics. We searched for the best linear characteristic of this cipher under the assumptions that a probability of any entry is not further from $1 / 2$ than $6 / 16$ and that the probability of a characteristic which relates one bit to one bit is not further from $1 / 2$ than $2 / 16$. Note that due to the relation between linear and differential characteristics, the searches are very similar; we actually modified the search program used in the differential case to search for the best linear characteristics with up to seven rounds, and those with the highest probabilities are given in Table 1

We can see that the probability of a 6 -round characteristic is bounded by $1 / 2 \pm 2^{-12.2}$, from which we can conclude that the probability of a 24-round characteristic is bounded by $1 / 2 \pm 2^{-45.8}$. The probability of a 28 -round characteristic is bounded by $1 / 2 \pm 2^{-52.2}$, and an attack based on it would require at least $2^{104}$ known plaintexts. Again, we wish to emphasize that all these figures are conservative lower bounds, and that the actual complexities of attacks are expected to be substantially higher.

Based on these figures we conjecture that the probability of the best 28-round linear differential is bounded by $1 / 2 \pm 2^{-50}$, so an attack would need at least $2^{100}$ blocks. Again, this is a very conservative estimate; we believe the real figure is over $2^{128}$ and that linear attacks are thus infeasible. We are working on more accurate figures; meantime the normal prudent practice of changing keys well before $2^{64}$ blocks have been encrypted will prevent linear attacks.

\subsection{Higher Order Differential Cryptanalysis}

It is well known that a $d$ th order differential of a function of nonlinear order $d$ is constant, and this can be exploited in higher order differential attacks [4,17/19]. The DES S-boxes all have nonlinear order 5 [18]. From this one would expect that the nonlinear order of the output bits after $r$ rounds is about $3^{r}$, with the 
maximum value of 127 reachable after five rounds. Therefore we are convinced that higher order differential attacks are not applicable to Serpent.

\subsection{Truncated Differential Cryptanalysis}

For some ciphers it is possible and advantageous to predict only the values of parts of the differences after each round. This notion, of truncated differential attacks, was introduced by Knudsen in 17. However, the method seems best applicable to ciphers where all operations are done on larger blocks of bits. Because of the strong diffusion over many rounds, we believe that truncated differential attacks are not applicable to Serpent.

\subsection{Related Keys}

As the key schedule uses rotations and S-boxes, it is highly unlikely that keys can be found that allow related key attacks [8,1516]. Moreover, different rounds of Serpent use different S-boxes, so even if related keys were found, related-key attacks would not be applicable.

Serpent has none of the simpler vulnerabilities that can result from exploitable symmetries in the key schedule: there are no weak keys, semi-weak keys, equivalent keys, or complementation properties.

\subsection{Other Attacks}

Davies' attack [12,13] and the Improved Davies attack [6] are not applicable, since the S-boxes are invertible, and no duplications of data bits are applied.

As far as we know, neither statistical cryptanalysis 22 nor partitioning cryptanalysis [14] provides a less complex attack than differential or linear cryptanalysis.

\subsection{Fault Analysis}

We have not been concerned in this design to build in any particular protection against attacks based on induced faults [3,10,11]. If an attacker can progressively remove the machine instructions by which this cipher is implemented, or progressively destroy selected gates, or progressively modify the bits of the key register, then he can clearly extract the key. We tend to the view that an attacker with the ability to inspect or modify the implementation detail will have many attacks based not just on compromising keys but on subverting protocols, extracting plaintext directly and so on [2]. The mechanisms required to protect against such attacks are largely independent of the design of any block cipher used [1], and are thus beyond the scope of this work. 


\section{Performance}

We implemented this cipher on a $133 \mathrm{MHz}$ Pentium/MMX processor. A 32round bitslice (unoptimized) implementation (available online from the authors' web pages) gave speeds which are only slightly slower than DES: it encrypted $8,976,157$ bits per second, while the best optimized DES implementation (Eric Young's Libdes) encrypts 9,824,864 bits per second on the same machine.

The performance of the cipher on other processors in bitslice mode should be only slightly slower than the standard implementation of DES. When coded in assembly language this cipher might be even faster than DES. It takes somewhat over 2000 instructions to encrypt 128 bits versus typically 700 instructions to encrypt 64 bits in DES. The reason our cipher is not $50 \%$ slower is that it has been designed to make good use of pipelining.

The instruction count is based on the observation that a gate circuit of any of the $4 \times 4$ S-boxes requires between 19 and 28 gates on the Pentium, between 18 and 28 on MMX (using only MMX instructions), and between 18 and 25 on the DEC Alpha (the numbers vary due to the different sets of instructions, which are detailed in the appendix). MMX has the additional advantage that it can operate on 64-bit words, or alternatively on two 32-bit words at once (so two encryptions can be done in parallel using the same or different keys). It is also implemented with greater parallelism on some recent chips (e.g. the Pentium II). On the other hand, it does not have rotate operations, so rotates require four instructions (copy, shift left, shift right, and OR).

It is also worth remarking that if this cipher is adopted as the Advanced Encryption Standard, and chip makers wish to support high speed implementation, then it may not be necessary to add a hardware encryption circuit to the CPU. It would be sufficient to add what we call the 'BITSLICE instruction'. This executes an arbitrary boolean function on four registers under the control of a truth table encoded in a (64-bit) fifth register. We estimate that the cost of implementing this on an $n$-bit processor will be only about $100 n$ gates, and it would have many uses other than cryptography (an example would be image processing). If supported, one BITSLICE instruction would replace most of the instructions in each round, and Serpent would become two or three times faster than DES.

It is also worth noting that hardware implementations of the cipher can iteratively apply one round at a time, although the S-boxes in each round are different. The trick is similar to the BITSLICE instruction: the designers of the hardware can design the round function to get a description of the S-boxes as a parameter in some register, and compute the S-boxes according to this description. This trick crucially reduces the number of gates required for the hardware implementation of the cipher. An estimate of the gate count will be provided in the full AES submission. 


\section{Other Variants}

As we remarked above, there are two ways in which the block size can be doubled:

1. increase the word length (in the bitslice implementation) from 32 to 64 bits (or more);

2. Use the round-function as the $F$-function in a Feistel construction.

If both of these are done, then the block size will be quadrupled.

These variants might require other modifications of the cipher, such as modifications in the rotation constants. We believe that these variants are secure (or can easily be made so). Work on them is ongoing.

\section{Conclusion}

We have presented a cipher which we have engineered to satisfy the AES requirements. It is about as fast as DES, and conjectured to be as secure as three-key triple DES. Its security is partially based on the reuse of the thoroughly studied components of DES, and thus can draw on the wide literature of block cipher cryptanalysis published in the last decade. Its performance comes from allowing an efficient bitslice implementation on a range of processors, including the market leading Intel/MMX and compatible chips.

This is still a preliminary design and may change between the time of writing and the final AES submission. Readers are invited to attack the cipher, to test implementations in various environments, and to report any interesting findings to the authors. A patent application has been filed, but it is our intention to grant a worldwide royalty-free license for conforming implementations in the event that this cipher is adopted as the Advanced Encryption Standard.

Finally, up to date information on Serpent, including the latest revision of the paper, can be found on the authors' home pages:

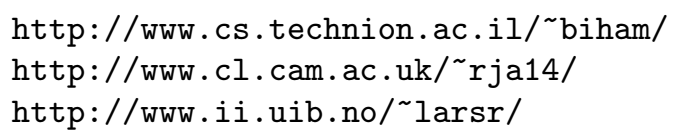

\section{Acknowledgments}

The first author was supported by Intel Corporation during a visit to Cambridge in September 1997 while much of this work was done; and the name of the cipher was suggested by Gideon Yuval (see Amos 5.19).

\section{References}

1. DG Abraham, GM Dolan, GP Double, JV Stevens, "Transaction Security System", in IBM Systems Journal v 30 no 2 (1991) pp 206-229 
2. RJ Anderson, MG Kuhn, "Tamper Resistance - a Cautionary Note", in The Second USENIX Workshop on Electronic Commerce Proceedings (Nov 1996) pp $1-11$

3. RJ Anderson, MG Kuhn, "Low Cost Attacks on Tamper Resistant Devices", to appear in proceedings of Security Protocols 97

4. E Biham, 'Higher Order Differential Cryptanalysis', unpublished paper, 1994

5. E Biham, How to Forge DES-Encrypted Messages in $2^{28}$ Steps, Technical Report CS884, Technion, August 1996

6. E Biham, A Biryukov, "An Improvement of Davies' Attack on DES", in Journal of Cryptology v 10 no 3 (Summer 97) pp 195-205

7. E Biham, A Shamir, 'Differential Cryptanalysis of the Data Encryption Standard' (Springer 1993)

8. E Biham, "New Types of Cryptanalytic Attacks Using Related Keys", in Journal of Cryptology v 7 (1994) no 4 pp 229-246

9. E Biham, "A Fast New DES Implementation in Software", in Fast Software Encryption - 4th International Workshop, FSE '97, Springer LNCS v 1267 pp 260-271

10. E Biham, A Shamir, "Differential Fault Analysis of Secret Key Cryptosystems", in Advances in Cryptology - Crypto 97, Springer LNCS v 1294 pp 513-525

11. D Boneh, RA DeMillo, RJ Lipton, "On the Importance of Checking Cryptographic Protocols for Faults", in Advances in Cryptology - Eurocrypt 97, Springer LNCS v 1233 pp 37-51

12. DW Davies, 'Investigation of a Potential Weakness in the DES Algorithm', private communication (1987)

13. D Davies, Murphy, "Pairs and Triplets of DES S Boxes", in Journal of Cryptology v 8 no 1 (1995) pp 1-25

14. C Harpes, JL Massey, "Partitioning Cryptanalysis", in Fast Software Encryption - 4th International Workshop, FSE '97, Springer LNCS v 1267 pp 13-27

15. J Kelsey, B Schneier, D Wagner, "Key-Schedule Cryptanalysis of IDEA, GDES, GOST, SAFER and Triple-DES", in Advances in Cryptology - Crypto 96, Springer LNCS v 1109 pp 237-251

16. LR Knudsen, "Cryptanalysis of LOKI91", in Advances in Cryptology - Auscrypt'92 Springer LNCS

17. LR Knudsen, "Truncated and Higher-Order Differentials", in Fast Software Encryption - 2nd International Workshop, FSE '94, Springer LNCS v 1008 pp 196-211

18. L.R. Knudsen, Block Ciphers - Analysis, Design and Applications, Ph.D. Thesis, Aarhus University, Denmark, 1994.

19. X.J. Lai, 'Higher Order Derivative and Differential Cryptanalysis', in Communication and Cryptography, Two Sides of one tapestry, R. Blahut (editor), Kluwer Academic Publishers, 1994

communication, September 30, 1993.

20. M Matsui, "Linear Cryptanalysis Method for DES Cipher", in Advances in Cryptology - Eurocrypt 93, Springer LNCS v 765 pp 386-397

21. RSA Data Security Inc., www.rsa.com

22. S Vaudenay, "An Experiment on DES Statistical Cryptanalysis", in 3rd ACM Conference on Computer and Communications Security, March 14-16, 96, New Delhi, India; proceedings published by ACM pp 139-147 


\section{A Appendix}

\section{A.1 The Initial Permutation IP:}

$\begin{array}{rrrrrrrrrrrrrrrr}0 & 32 & 64 & 96 & 1 & 33 & 65 & 97 & 2 & 34 & 66 & 98 & 3 & 35 & 67 & 99 \\ 4 & 36 & 68 & 100 & 5 & 37 & 69 & 101 & 6 & 38 & 70 & 102 & 7 & 39 & 71 & 103 \\ 8 & 40 & 72 & 104 & 9 & 41 & 73 & 105 & 10 & 42 & 74 & 106 & 11 & 43 & 75 & 107 \\ 12 & 44 & 76 & 108 & 13 & 45 & 77 & 109 & 14 & 46 & 78 & 110 & 15 & 47 & 79 & 111 \\ 16 & 48 & 80 & 112 & 17 & 49 & 81 & 113 & 18 & 50 & 82 & 114 & 19 & 51 & 83 & 115 \\ 20 & 52 & 84 & 116 & 21 & 53 & 85 & 117 & 22 & 54 & 86 & 118 & 23 & 55 & 87 & 119 \\ 24 & 56 & 88 & 120 & 25 & 57 & 89 & 121 & 26 & 58 & 90 & 122 & 27 & 59 & 91 & 123 \\ 28 & 60 & 92 & 124 & 29 & 61 & 93 & 125 & 30 & 62 & 94 & 126 & 31 & 63 & 95 & 127\end{array}$

\section{A.2 The Final Permutation FP:}

$\begin{array}{rrrrllllllrrrrrr}0 & 4 & 8 & 12 & 16 & 20 & 24 & 28 & 32 & 36 & 40 & 44 & 48 & 52 & 56 & 60 \\ 64 & 68 & 72 & 76 & 80 & 84 & 88 & 92 & 96 & 100 & 104 & 108 & 112 & 116 & 120 & 124 \\ 1 & 5 & 9 & 13 & 17 & 21 & 25 & 29 & 33 & 37 & 41 & 45 & 49 & 53 & 57 & 61 \\ 65 & 69 & 73 & 77 & 81 & 85 & 89 & 93 & 97 & 101 & 105 & 109 & 113 & 117 & 121 & 125 \\ 2 & 6 & 10 & 14 & 18 & 22 & 26 & 30 & 34 & 38 & 42 & 46 & 50 & 54 & 58 & 62 \\ 66 & 70 & 74 & 78 & 82 & 86 & 90 & 94 & 98 & 102 & 106 & 110 & 114 & 118 & 122 & 126 \\ 3 & 7 & 11 & 15 & 19 & 23 & 27 & 31 & 35 & 39 & 43 & 47 & 51 & 55 & 59 & 63 \\ 67 & 71 & 75 & 79 & 83 & 87 & 91 & 95 & 99 & 103 & 107 & 111 & 115 & 119 & 123 & 127\end{array}$

\section{A.3 The Linear Transformation:}

For each output bit of this transformation, we describe the list of input bits whose parity becomes the output bit. In each row we describe four output bits, which later enter the same S-box in the next round. The bits are listed from 0 to 127 .

\begin{tabular}{|c|c|c|c|c|c|c|c|c|c|c|c|c|c|c|c|c|c|c|c|}
\hline & & & & 83 & 94 & & & & & & & & & & & & & & \\
\hline 20 & 566 & 60 & 74 & 87 & 98 & $109\}$ & $\{1$ & 76 & 118 & $\{2$ & 6 & 3 & & 44 & 8 & $88\}$ & $\{40$ & & \\
\hline & 06 & 64 & 78 & 91 & 102 & $113\}$ & $\{5$ & 80 & $122\}$ & & 10 & 7 & & & & $92\}$ & 44 & 98 & \\
\hline & 46 & 68 & 82 & 95 & 06 & $117\}$ & $\{9$ & 84 & & $\{10$ & 14 & & & & & & 48 & 102 & \\
\hline & & & 36 & 99 & 10 & $121\}$ & $\{2$ & 13 & & & 18 & & & & 2 & & & 06 & \\
\hline & & & 90 & 103 & 14 & $125\}$ & & 17 & & & 2 & & & & & & & 110 & \\
\hline & & & 80 & 94 & 07 & 118 & & 21 & & & 6 & & & & & & & 114 & \\
\hline & & & 84 & 98 & & & & 5 & & & & 7 & & & & & & 118 & \\
\hline & & & 88 & 102 & & & & 9 & 104 & & & & & & & & & 122 & \\
\hline & & & 88 & 92 & & & & 3 & & & & & & & & & 1. & 126 & \\
\hline & & & & 96 & & & & 7 & & & 42 & 9 & & & & & & 15 & \\
\hline & & & & 100 & 4 & & & 1 & 11 & & 42 & 6 & & 9 & 74 & & & 19 & \\
\hline & & & & & & & & 45 & 120 & & 46 & & & & 8 & & & 23 & \\
\hline & & & & 108 & 22 & & & 49 & 124] & & 8 & & & & & & & 7 & \\
\hline & & & 8 & 112 & 26 & & & 2 & & & 12 & & & & & & 18 & 1 & \\
\hline & & & & 76 & 2 & 116 & & 46 & & & 16 & & & & & & & 5 & \\
\hline & & & & 80 & 6 & 120 & & 0 & & & 20 & & & & & & & 9 & \\
\hline & & & & 84 & 120 & 124 & & & & & 24 & & & & & & & 3 & \\
\hline & & & & 49 & 88 & 124 & & & & & 28 & & & & & & & & \\
\hline & & & & 42 & 53 & 92 & & & & & & & & & & & & & \\
\hline & & & & 46 & & $96\}$ & & 66 & 77\} & & & & 82 & 89 & 95 & $110\}$ & & 55 & \\
\hline
\end{tabular}




\begin{tabular}{|c|c|c|c|c|c|c|c|c|c|c|c|c|c|c|c|c|}
\hline $\begin{array}{lll}8 & 12 & 26\end{array}$ & 39 & 50 & 61 & $100\}$ & $\{28$ & 70 & $81\}$ & $\{32$ & 40 & 82 & 86 & 93 & 99 & $114\}\{46$ & 59 & $120\}$ \\
\hline$\left\{\begin{array}{lll}12 & 16 & 30\end{array}\right.$ & 43 & 54 & 65 & $104\}$ & $\{32$ & 74 & $85\}$ & $\{36$ & 90 & 103 & 118 & & & \}$\{50$ & 63 & $124\}$ \\
\hline $\begin{array}{lll}6 & 20 & 34\end{array}$ & 47 & 58 & 69 & $108\}$ & $\{36$ & 78 & $89\}$ & $\{40$ & 94 & 107 & 122 & & & \}$\{0$ & 54 & $67\}$ \\
\hline 02438 & 51 & 62 & 73 & $112\}$ & $\{40$ & 82 & $93\}$ & $\{44$ & 98 & 111 & 126 & & & $\{4$ & 58 & $71\}$ \\
\hline 28 & 55 & 66 & 77 & $116\}$ & $\{44$ & 86 & $97\}$ & $\{2$ & 48 & 102 & 115 & & & $\{8$ & 62 & $75\}$ \\
\hline 32 & 59 & 70 & 81 & $120\}$ & $\{48$ & 90 & $101\}$ & $\{6$ & 52 & 106 & 110 & & & $\{12$ & 66 & $79\}$ \\
\hline 6 & 63 & 74 & 85 & $124\}$ & $\{52$ & 94 & $105\}$ & $\{10$ & 56 & 110 & 123 & & & $\{16$ & 70 & $83\}$ \\
\hline 10 & 54 & 67 & 78 & $89\}$ & $\{56$ & 98 & $109\}$ & $\{14$ & 60 & 114 & 127 & & & \}$\{20$ & 74 & $87\}$ \\
\hline & 58 & 71 & 82 & $93\}$ & $\{60$ & 102 & 113\} & $\{3$ & 18 & 72 & 114 & 118 & 125 & \}$\{24$ & 78 & $91\}$ \\
\hline & 62 & 75 & 86 & $97\}$ & $\{64$ & 106 & $117\}$ & $\{1$ & 7 & 22 & 76 & 118 & 122 & \}$\{28$ & 82 & $95\}$ \\
\hline & 66 & 79 & 90 & $101\}$ & $\{68$ & 110 & $121\}$ & $\{5$ & 11 & 26 & 80 & 122 & 126 & \}$\{32$ & 86 & $99\}$ \\
\hline
\end{tabular}

\section{A.4 S-Boxes}

Here are the S-boxes $S_{0}$ through $S_{31}$ (each on a separate line):

$\begin{array}{llllllllllllllll}14 & 4 & 13 & 1 & 2 & 15 & 11 & 8 & 3 & 10 & 6 & 12 & 5 & 9 & 0 & 7\end{array}$ $\begin{array}{llllllllllllllll}0 & 15 & 7 & 4 & 14 & 2 & 13 & 1 & 10 & 6 & 12 & 11 & 9 & 5 & 3 & 8\end{array}$ $\begin{array}{llllllllllllllll}4 & 1 & 14 & 8 & 13 & 6 & 2 & 11 & 15 & 12 & 9 & 7 & 3 & 10 & 5 & 0\end{array}$ $\begin{array}{llllllllllllllll}15 & 12 & 8 & 2 & 4 & 9 & 1 & 7 & 5 & 11 & 3 & 14 & 10 & 0 & 6 & 13\end{array}$ $\begin{array}{llllllllllllllll}15 & 1 & 8 & 14 & 6 & 11 & 3 & 4 & 9 & 7 & 2 & 13 & 12 & 0 & 5 & 10\end{array}$ $\begin{array}{llllllllllllllll}3 & 13 & 4 & 7 & 15 & 2 & 8 & 14 & 12 & 0 & 1 & 10 & 6 & 9 & 11 & 5\end{array}$ $\begin{array}{llllllllllllllll}0 & 14 & 7 & 11 & 10 & 4 & 13 & 1 & 5 & 8 & 12 & 6 & 9 & 3 & 2 & 15\end{array}$ $\begin{array}{llllllllllllllll}13 & 8 & 10 & 1 & 3 & 15 & 4 & 2 & 11 & 6 & 7 & 12 & 0 & 5 & 14 & 9\end{array}$ $\begin{array}{llllllllllllllll}10 & 0 & 9 & 14 & 6 & 3 & 15 & 5 & 1 & 13 & 12 & 7 & 11 & 4 & 2 & 8\end{array}$ $\begin{array}{llllllllllllllll}13 & 7 & 0 & 9 & 3 & 4 & 6 & 10 & 2 & 8 & 5 & 14 & 12 & 11 & 15 & 1\end{array}$ $\begin{array}{lllllllllllllllll}13 & 6 & 4 & 9 & 8 & 15 & 3 & 0 & 11 & 1 & 2 & 12 & 5 & 10 & 14 & 7\end{array}$ $\begin{array}{llllllllllllllll}1 & 10 & 13 & 0 & 6 & 9 & 8 & 7 & 4 & 15 & 14 & 3 & 11 & 5 & 2 & 12\end{array}$ $\begin{array}{llllllllllllllll}7 & 13 & 14 & 3 & 0 & 6 & 9 & 10 & 1 & 2 & 8 & 5 & 11 & 12 & 4 & 15\end{array}$ $\begin{array}{llllllllllllllll}13 & 8 & 11 & 5 & 6 & 15 & 0 & 3 & 4 & 7 & 2 & 12 & 1 & 10 & 14 & 9\end{array}$ $\begin{array}{llllllllllllllll}10 & 6 & 9 & 0 & 12 & 11 & 7 & 13 & 15 & 1 & 3 & 14 & 5 & 2 & 8 & 4\end{array}$ $\begin{array}{llllllllllllllll}3 & 15 & 0 & 6 & 10 & 1 & 13 & 8 & 9 & 4 & 5 & 11 & 12 & 7 & 2 & 14\end{array}$ $\begin{array}{llllllllllllllll}2 & 12 & 4 & 1 & 7 & 10 & 11 & 6 & 8 & 5 & 3 & 15 & 13 & 0 & 14 & 9\end{array}$ $\begin{array}{llllllllllllllll}14 & 11 & 2 & 12 & 4 & 7 & 13 & 1 & 5 & 0 & 15 & 10 & 3 & 9 & 8 & 6\end{array}$ $\begin{array}{llllllllllllllll}4 & 2 & 1 & 11 & 10 & 13 & 7 & 8 & 15 & 9 & 12 & 5 & 6 & 3 & 0 & 14\end{array}$ $\begin{array}{llllllllllllllll}11 & 8 & 12 & 7 & 1 & 14 & 2 & 13 & 6 & 15 & 0 & 9 & 10 & 4 & 5 & 3\end{array}$ $\begin{array}{llllllllllllllll}12 & 1 & 10 & 15 & 9 & 2 & 6 & 8 & 0 & 13 & 3 & 4 & 14 & 7 & 5 & 11\end{array}$ $\begin{array}{llllllllllllllll}10 & 15 & 4 & 2 & 7 & 12 & 9 & 5 & 6 & 1 & 13 & 14 & 0 & 11 & 3 & 8\end{array}$ $\begin{array}{llllllllllllllll}9 & 14 & 15 & 5 & 2 & 8 & 12 & 3 & 7 & 0 & 4 & 10 & 1 & 13 & 11 & 6\end{array}$ $\begin{array}{llllllllllllllll}4 & 3 & 2 & 12 & 9 & 5 & 15 & 10 & 11 & 14 & 1 & 7 & 6 & 0 & 8 & 13\end{array}$ $\begin{array}{llllllllllllllll}4 & 11 & 2 & 14 & 15 & 0 & 8 & 13 & 3 & 12 & 9 & 7 & 5 & 10 & 6 & 1\end{array}$ $\begin{array}{llllllllllllllll}13 & 0 & 11 & 7 & 4 & 9 & 1 & 10 & 14 & 3 & 5 & 12 & 2 & 15 & 8 & 6\end{array}$ $\begin{array}{llllllllllllllll}1 & 4 & 11 & 13 & 12 & 3 & 7 & 14 & 10 & 15 & 6 & 8 & 0 & 5 & 9 & 2\end{array}$ $\begin{array}{llllllllllllllll}6 & 11 & 13 & 8 & 1 & 4 & 10 & 7 & 9 & 5 & 0 & 15 & 14 & 2 & 3 & 12\end{array}$ $\begin{array}{llllllllllllllll}13 & 2 & 8 & 4 & 6 & 15 & 11 & 1 & 10 & 9 & 3 & 14 & 5 & 0 & 12 & 7\end{array}$ $\begin{array}{llllllllllllllll}1 & 15 & 13 & 8 & 10 & 3 & 7 & 4 & 12 & 5 & 6 & 11 & 0 & 14 & 9 & 2\end{array}$ $\begin{array}{llllllllllllllll}7 & 11 & 4 & 1 & 9 & 12 & 14 & 2 & 0 & 6 & 10 & 13 & 15 & 3 & 5 & 8\end{array}$ $\begin{array}{llllllllllllllll}2 & 1 & 14 & 7 & 4 & 10 & 8 & 13 & 15 & 12 & 9 & 0 & 3 & 5 & 6 & 11\end{array}$ 


\title{
A.5 Lists of Relevant Instructions on Various Processors
}

The relevant instructions on the following processors are:

\author{
Pentium: AND, OR, XOR, NOT, rotate \\ MMX: $\quad$ AND, OR, XOR, NOT, ANDN, only shifts \\ Alpha: $\quad$ AND, OR, XOR, NOT, ANDN, ORN, XORN, only shifts
}

where the ANDN operation on $x$ and $y$ is $x \wedge(\neg y)$, the ORN operation is $x \vee(\neg y)$, and the XORN operation is $x \oplus(\neg y)$ (or equivalently $\neg(x \oplus y)$ ).

On MMX a rotate takes four instructions, while on an Alpha it takes three. On Pentium and MMX it might be necessary to copy some of the registers before use, as instructions have only two arguments; but some instructions can refer directly to memory. The Alpha instructions have 3 arguments (src1, src2 and destination), but cannot refer directly to memory.

\section{A.6 Historical Remarks}

Here we describe some design history. In our first design, the linear transformations were just bit permutations, which were applied as rotations of the 32-bit words in the bitslice implementation. In order to ensure maximal avalanche, the idea was to choose these rotations in a way that ensured maximal avalanche in the fewest number of rounds. Thus, we chose three rotations at each round: we used $(0,1,3,7)$ for the even rounds and $(0,5,13,22)$ for the odd rounds. The reason for this was that (a) rotating all four words is of course useless (b) a single set of rotations did not suffice for full avalanche (c) these sets of rotations have the property that no difference of pairs in either of them coincides with a difference either in the same set or the other set.

However, we felt that the avalanche was still slow, as each bit affected only one bit in the next round, and thus one active S-box affected only 2-4 out of the 32 S-boxes in the next round. As a result, we had to use 64 rounds, and the cipher was only slightly faster than triple-DES. So we moved to a more complex linear transformation; this improved the avalanche, and analysis showed that we could now reduce the number of rounds to 32 . We believe that the final result is a faster and yet more secure cipher.

We also considered "improving" the cipher by replacing the XOR operations by seemingly more complex operations, such as additions. We did not do this due to two major reasons: (1) Our analysis takes advantage of the independence between the bits in the XOR operation, as it allows us to describe the cipher in a standard way, and use the known kinds of security analysis. This analysis would not hold if the XOR operations were replaced; (2) in some other ciphers the replacement of XORs by additions (or other operations) has turned out to weaken the cipher, rather than strengthening it. 
238 Eli Biham, Ross Anderson, Lars R. Knudsen

\section{A.7 Reference Implementation}

An unoptimized reference C implementation is available from the authors' home pages. Note however that the cipher may still be modified in the future as it progresses through the AES selection process. 